\title{
Trauma and Life Events in Adults with Intellectual Disability
}

\author{
Sarah Wigham • Eric Emerson
}

Published online: 11 February 2015

(C) Springer International Publishing Switzerland 2015

\begin{abstract}
Exposure to adverse life events and environmental stressors (e.g. violence, poverty, sexual abuse) has been found to be high in intellectual disability populations. Notwithstanding resilience, for some people with intellectual disability, adverse life events and environmental stressors may lead to PTSD and may affect well-being in more diffuse but potentially still clinically impactful ways (e.g. challenging behaviour, obesity, substance misuse). Advances have occurred in the field of trauma in intellectual disability. More specifically, a small number of assessment measures have been developed for PTSD and a small number of studies evaluated treatment interventions such as cognitive behavioural therapy (CBT) and eye movement desensitisation and reprocessing (EMDR). Awareness has also increased regarding the potential life course effects on mental health of exposure to environmental stressors. While these developments are promising, the field warrants more research and development. This paper considers recent developments in knowledge on the psychological effects of exposure to adverse life events and environmental stressors in adults with intellectual disabilities.
\end{abstract}

Keywords Trauma $\cdot$ Life events $\cdot$ PTSD .

Intellectual disability · Assessment $\cdot$ Treatment .

Environmental stressors $\cdot$ Mental health

This article is part of the Topical Collection on Intellectual Disability

S. Wigham $(\bowtie)$

Institute of Neuroscience, Faculty of Medical Sciences,

Newcastle University, Henry Wellcome Building, Framlington Place,

Newcastle upon Tyne NE2 4HH, UK

e-mail: sarah.wigham@ncl.ac.uk

E. Emerson

Centre for Disability Research \& Policy, University of Sydney,

Sydney, Australia

\section{Introduction}

Exposure to environmental stressors such as poverty is higher among people with intellectual disabilities when compared to their non-disabled peers [1]. For example, narrative interviews with women with intellectual disabilities revealed experiences of domestic violence from childhood and continuing on into intimate relationships in adulthood [2•]; students with intellectual disabilities were exposed to more interpersonal abuse than typically developing peers, and [3] events at Winterbourne View, UK evidenced exposure to carer-perpetrated violence in institutional settings [4].

It is important to avoid a disempowering perspective that paternalistically frames this population group as past or future trauma victims [5-7] or to over-pathologise life experience and suggest that people with intellectual disability are not resilient $[8,9]$. However, the increased likelihood of being exposed to environmental stressors means people with intellectual disabilities are at a higher risk of having their resilience compromised and developing mental and physical health problems including PTSD $[1,10,11]$.

For the purposes of this paper, environmental stressors are defined as an over-arching category encompassing all potential life stressors including individual adverse life events. Within this framework, particular stressors will vary in terms of how traumatising a single exposure is, the pattern of exposure, duration and repetition. For example, the death of a child is rare but high impact, while poverty is a state characterised by exposure to various repeated lower impact stressors, the cumulative consequence of which can be damaging to health [12]. Although environmental stressors can impact on physical and mental health, this paper will focus on psychological effects or where physical conditions may be linked to psychological distress such as obesity.

The paper which is a narrative rather than systematic review will consider some recent issues pertaining to the effects 
on people with intellectual disabilities of exposure to adverse life events and environmental stressors, effects including PTSD and other diffuse but still clinically impactful influences on mental health. The paper will consider and evaluate recent literature published in the area including on treatment and assessment.

\section{Recent Research: Effects on People with ID of Exposure to Adverse Life Events and Environmental Stressors}

A number of recent studies have documented the extent to which people with intellectual disabilities (ID) are at increased risk of exposure to adverse life events and environmental stressors. These include common social determinants of poor health (e.g. poor housing, violence in the family, lower income, social isolation) among a large population-based sample of children with developmental delay [13•] and childhood abuse and neglect among juvenile offenders [14•]. In addition, in narrative interviews with women with intellectual disability, familial relationship patterns in childhood were described as characterised by violence, devaluation and rejection and seen to create a susceptibility to exploitative and abusive intimate relationships in adulthood [2•].

Two studies extended the evidence base for correlational relationships between adverse life events and reduced psychological well-being in people with intellectual disabilities by using prospective designs. A unidirectional relationship was found between negative life events as measured using the Bangor Life Events Schedule for Intellectual Disabilities (a 38-item informant measure of life events) and psychological and behavioural problems at follow-up [15]. Similarly, negative life events were found significantly predictive of psychological trauma assessed 6 months later by a measure of trauma developed specifically for people with intellectual disabilities, the Lancaster and Northgate Trauma Scales (LANTS; [16]).

\section{Disorders Associated with Exposure to Adverse Life Events}

PTSD in Intellectual Disabilities: Conceptual Issues

The conceptualisation and diagnosis of trauma and PTSD in general population cohorts continue to be a contested area. For example, current debated issues include whether depression and PTSD are distinct outcomes from experiencing trauma or overlapping constructs [17]. There are similar, if not greater, conceptual problems relating to the conceptualisation and diagnosis of trauma in intellectual disability cohorts. For example, it remains unclear whether the general population conceptualisation of PTSD has some limitations in terms of validity when applied to people with intellectual disabilities [18].
Nevertheless, much can probably be learned from work already done in relation to general population cohorts especially in relation to issues that are transferrable but not specific to intellectual disability populations [19].

One area of much debate is the identification of a specific criterion A1 event for a diagnosis of PTSD. Assessment and treatment of PTSD are focussed around this event and associated symptoms (e.g. nightmares of the event). However, the definition of such an event and whether to exclude the requirement completely from DSM criteria is a debated issue. Naming an event is not always possible in general population cohorts, raising some challenges for assessment and treatment of PTSD which are therefore not necessarily unique to intellectual disability populations. For example, secrecy, stigma and shame may mean interpersonal trauma like sexual abuse is not always articulated and may remain so for years [20]. In addition to this, cognitive and communication difficulties in intellectual disability populations may limit abilities to articulate a criterion A1 event and describe symptoms pertaining to the event (e.g. flashbacks, nightmares). This may mean exclusion from access to a diagnosis and subsequent treatment. It has been debated whether the criterion A1 event be excluded completely from the PTSD symptom criteria [21] or whether the definition of what constitutes a criterion A1 event be widened or narrowed [22-24]. From a developmental perspective, a different model of trauma has been suggested for youth with a study of a cohort of 18-19-year-olds finding that some adverse childhood experiences would not meet the threshold for criterion A1 events and the likelihood of experiencing multiple events challenging the validity of tying a diagnosis to the effects of one event [25]. These issues are particularly relevant to intellectual disability populations.

\section{Assessment and Treatment of PTSD in ID: What We Knew 12 Months Ago}

A measure of trauma developed specifically for people with intellectual disabilities is the Lancaster and Northgate Trauma Scale (LANTS). The measure has 29-item self-report and 43item informant-report versions. Preliminary findings show promising psychometric properties in people with mild to moderate intellectual disabilities [18]. Methods have been used to increase the accessibility of trauma assessments, and where an individual is unable to articulate a trigger event due to cognitive and communication difficulties, then, biographical timelines of events and a trauma history have been constructed with information gathered from several other sources [26-28].

The empirical evidence of treatment for PTSD in people with intellectual disabilities including eye movement desensitisation and reprocessing (EMDR), trauma focussed cognitive behavioural therapy (CBT) and psychodynamic interventions has been reviewed and found small but promising 
[29]. EMDR is suggested to be an effective intervention for other conditions resulting from trauma (e.g. depression) though the underlying mechanisms are not well understood [30]. EMDR may be particularly useful with some people with intellectual disabilities as it does not require identification of a traumatic event or articulation regarding the cognitive and emotional aspects of the event in order to have therapeutic effect [26]. Where a traumatic event cannot be articulated by an individual but can be reported by a third party even if current behaviour cannot obviously be linked with past events, then, current concerns, difficult emotions and affective states can be addressed instead to reduce arousal levels and stabilise mood [26]. Adaptations to EMDR interventions to increase their accessibility have included splitting the trauma event into smaller components, more verbal input by the therapist, and the use of bilateral tactile and auditory instead of visual stimulation [26].

\section{Assessment and Treatment of PTSD in ID: Recent Studies}

The Impact of Event Scale-Intellectual Disabilities (IES-IDs) [31] is a 22-item self-report screening measure newly developed from the Impact of Events Scale, a widely used scale to screen for PTSD in the general population. Respondents are asked to name an event and then questions asked about current feelings and behaviours with regard to the event. Completed by people with mild intellectual disabilities, the psychometric properties of the IES-ID were promising, suggesting it to be a useful self-report screening measure for people with mild to moderate intellectual disabilities.

A case study design of five Norwegian adults with moderate to severe intellectual disabilities demonstrated that diagnosis of PTSD was possible by using information gathered from third parties (e.g. family members, carers and staff), case notes, interviews and the informant-report Lancaster and Northgate Trauma Scales. The authors note that prior to the diagnosis of PTSD, other diagnoses given included psychosis, personality disorder, depression and challenging behaviour [32-]. An accurate diagnosis can help staff caring for the person have more insight into the individual's presentation and how best to care for them (e.g. that their anxiety is due to PTSD) [32•]. For treatment, psychotherapy was used, and milieu therapy with attention to and sensitive interpretation of upsetting triggers promoting the regulation of mood [32•].

\section{Other Disorders Associated with Exposure to Adverse Life Events and Environmental Stressors}

It is beyond the remit of this paper to look at all potential effects of trauma on mental health in any great depth. However, it is useful to highlight some general issues pertinent to reactive mental health conditions.
Trauma and Challenging Behaviour

Anger and aggression can be symptoms of PTSD in the general population [33, 34], and so where challenging behaviour does occur in intellectual disability populations it may be the result of trauma [28, 35]. As evidence regarding the presentation of trauma in people with moderate to severe intellectual disabilities is particularly limited, PTSD and other traumarelated psychological distress may simply be categorised as challenging behaviour or misdiagnosed as psychosis [32•]. In a sample of women with borderline personality disorder and histories of severe interpersonal trauma, emotional processing was impaired and affected working memory such that emotional information was more distracting compared to healthy controls [36]. Such neurological level changes have implications for the presentation of traumatised people with intellectual disabilities who may acquire a label of being difficult or challenging if working memory for the task at hand is impaired in situations of high emotional valence.

People with intellectual disabilities may lack the resources to create lives for themselves which facilitate physical or psychological avoidance of reminders of past trauma (e.g. by having limited opportunities for employment or independent living). Living in an institutional environment means an individual is less likely to have control over their environment in order to avoid upsetting triggers related to past trauma such that their care potentially becomes a source of environmental stress. For example, movement to out of area placements as a result of challenging behaviour may add to an individual's stress and also reduce opportunities for information sharing (e.g. of past traumas and current triggers) by disrupting support systems with families, friends and services [37].

A label of challenging behaviour is stigmatising and has implications for quality of life, mental and physical health and relationships. Where behaviour is framed as problematic or challenging, an individual may be viewed only as a perpetrator rather than having been a victim in the past, and so, management may be more punitive than therapeutic for example with seclusion, restraints [38, 39], being moved to out of area placements [37] and medication. Anti-psychotics are used extensively with people with intellectual disabilities [40], often controversially to manage behaviour where no underlying diagnosis such as PTSD has been identified and with limited evidence base for their clinical or cost-effectiveness [41, 42]. Medication also has side effects [43], and its use may mean that potentially effective treatment is delayed [44•].

\section{Trans-Diagnostic Effects of Trauma}

Apart from PTSD, adverse life events and environmental stressors can have other trans-diagnostic and clinically impactful effects on health. Trauma from abuse and neglect was found to have detrimentally affected the development 
and then transition to independence of youth with intellectual disabilities [45] and increased the risk of sexual and violent offending [14•]. Childhood adversity has been linked to depression, substance misuse and obesity in adulthood in the general population [46]. Childhood abuse was found associated with obesity-related inflammation in middle-aged women in the general population [47], and with obesity high in intellectual disability populations [48], the link with past trauma may warrant consideration. Evidence also suggests that where access is possible, people with intellectual disabilities are at a high risk of substance misuse $[49,50]$ which may be a coping strategy for emotional problems [51]. Major depressive disorder was found to be higher in a Dutch cohort of individuals aged over 50 years old than in the general population though the authors noted limitations in the ability to validly assess and diagnose depression in ID groups [52]; the risk of suicide has also been highlighted in this group though again screening is compromised by the lack of valid and reliable tools [53, 54].

Fourteen generic measures of mental health were identified in a recent review [55]. Although this is not an insubstantial number, the authors note the importance of a range of measures being available to facilitate assessing different developmental levels, ages and formats for different responders [55]. Reviews of measures of anxiety and depression describe several assessment tools available for this population group though note limited evidence of reliability, validity, sensitivity and specificity $[56,57]$. Having reliable and valid assessments for specific mental health disorders is important, and use of these as screening tools can reduce the chances of psychological pathology with mental health diagnoses made early and accurately avoiding chronicity, imperviousness to treatment, complication and misdiagnosis [58].

Cognitive behavioural interventions have been found effective [59-62] and acceptable [63] across a number of psychological conditions in people with mild intellectual disabilities. Mindfulness-based cognitive behavioural therapy (CBT) was found helpful in adults with intellectual disabilities, anxiety and depression [64]. The lack of an evidence base for treatment interventions for women with mental health problems and possible histories of sexual abuse has been noted and implicated in their lengthy stays in high-security forensic settings [44•].

\section{Trauma and Organisational Issues}

Societal feedback at micro level (e.g. interpersonal reactions to feelings of guilt) and macro level (e.g. organisational reactions to anger) may be important mediating factors in the development and maintenance of trauma [65]. Given that reactions to trauma develop as an interaction with variables in the social landscape and are not just a process located within the individual, it is important to consider environment factors
[66]. Trauma-informed care (TIC) considers the impact of organisational structure and procedures and staff on traumatised individuals [67]. The aim of trauma-informed care services is that organisations be more attuned to the possibility of trauma in a person's past and its role in the aetiology of current presentation. TIC promotes awareness raising to increase staff insight and understanding regarding trauma, so the possibility of past trauma is held in mind [68].

The model proposes creating environments which promote trust, a sense of safety, boundaries, empowerment and choice, and introduction of the model to intellectual disability services has recently been described [67]. The approach requires training and the creation of a particular organisational culture and service delivery which has implications for resources and care of staff $[67,69]$. This may be difficult in environments where there is a lack of opportunity to develop independence, low staff to service user ratio, high staff turnover and limited opportunities for training [67]. Thorough information gathering for biographical timelines and identification of trauma triggers is very promising for the development of appropriate interventions for people with moderate to severe intellectual disabilities but is time intensive, and working at this level day to day has implications for resources and education of carers [26]. Gathering and recording information on past life events (e.g. by using the BLESID life events schedule) [15] may be a useful routine practice for services especially if individuals are moved between placements frequently [37]. An example of a training course is 'Who's Challenging Who' which aimed to change staff attitudes and increase empathy for challenging behaviour [70].

\section{Conclusions}

This narrative review has highlighted the relatively higher exposure to environmental stressors of people with intellectual disabilities and the possible trans-diagnostic effects of these experiences on mental health. When adverse psychological effects of environmental stressors are experienced by people with intellectual disabilities, the evidence base underpinning their options for valid and reliable diagnosis and intervention is more limited compared to that for the general population [19]. However, debates around the conceptualisation of trauma in general population cohorts are of relevance to intellectual disability populations, including the definition and requirement of a criterion A1 event for a diagnosis of PTSD and potential compromises to diagnosis and intervention if an event cannot be articulated. In this context, the challenges to developing assessments and interventions for people with moderate to severe intellectual disabilities have begun to be explored creatively with sensitive and flexible adaptation of existing assessments and interventions and focus on addressing present arousal and affective states even where a link to 
past trauma cannot be articulated by the individual (e.g. using bilateral auditory and touch stimulation in EMDR), and these are definitely promising.

The paper highlights recent research focusing on the value of service provider level consideration and facilitation of openness to the possibility of trauma in the aetiology of diffuse but clinically impactful presentations such as challenging behaviour, obesity and depression. The articles in the review suggest the need for further work crystallising conceptualizations of trauma and its trans-diagnostic effects in people with ID in a context of organisations sensitive to the effects of past life events and environmental stressors.

An important area for future research is the further development and psychometric validation of assessment measures specifically for people with intellectual disabilities. There is utility in having brief measures which are quick to complete in screening and research settings plus more in-depth measures for clinical detail and diagnosis, with the latter being important for acquiring services and informing other people's reactions $[32 \cdot 55]$.

\section{Compliance with Ethics Guidelines}

Conflict of Interest Sarah Wigham and Eric Emerson declare that they have no conflict of interest.

Human and Animal Rights and Informed Consent This article does not contain any studies with human or animal subjects performed by any of the authors.

\section{References}

Papers of particular interest, published recently, have been highlighted as:

- Of importance

1. Emerson E, Hatton C. Mental health of children and adolescents with intellectual disabilities in Britain. Br J Psychiatry. 2007;191(6):493-9.

2. Pestka K, Wendt S. Belonging: women living with intellectual disabilities and experiences of domestic violence. Disabil Soc. 2014;29(7):1031-45. Describes narrative interviews with women with intellectual disabilities about experiences of childhood abuse and a legacy of domestic violence in adulthood.

3. Reiter S, Bryen DN, Shacher I. Adolescents with intellectual disabilities as victims of abuse. J Intellect Disabil. 2007;11(4):371-87.

4. Oakes P. Crash: what went wrong at Winterbourne View? J Intellect Disabil. 2012;16(3):155-62.

5. Ward N. Care ethics and carers with learning disabilities: a challenge to dependence and paternalism. Ethics Soc Welf. 2011;5(2):168-80.

6. Ditchman N, Werner S, Kosyluk K, Jones N, Elg B, Corrigan PW. Stigma and intellectual disability: potential application of mental illness research. Rehabil Psychol. 2013;58(2):206-16.
7. Northway R, Bennett D, Melsome M, et al. Keeping safe and providing support: a participatory survey about abuse and people with intellectual disabilities. J Policy Pract Intellect Disabil. 2013;10(3): 236-44.

8. Goodley D. Empowerment, self-advocacy and resilience. J Intellect Disabil. 2005;9(4):333-43.

9. McRitchie R, McKenzie K, Quayle E, Harlin M, Neumann K. How adults with an intellectual disability experience bereavement and grief: a qualitative exploration. Death Stud. 2014;38(3):179-85.

10. Emerson E. Poverty and people with intellectual disabilities. Ment Retard Dev Disabil Res Rev. 2007;13(2):107-13.

11. Emerson E. Commentary: childhood exposure to environmental adversity and the well-being of people with intellectual disabilities. J Intellect Disabil Res. 2013;57(7):589-600.

12. World Health Organization. Closing the gap in a generation: health equity through action on the social determinants of health. Final report of the Commission on the Social Determinants of Health. Geneva: World Health Organization; 2008.

13. Emerson E, Brigham P. Exposure of children with developmental delay to social determinants of poor health: cross-sectional case record review study. Child Care Health Dev. 2014. doi:10.1111/ cch.12144. Supports the value of family level early intervention by demonstrating negative physical and behavioural effects due to the increased exposure to social determinants of health of a large population based sample of children with developmental delay.

14. van der Put CE, Asscher JJ, Wissink IB, Stams GJJM. The relationship between maltreatment victimisation and sexual and violent offending: differences between adolescent offenders with and without intellectual disability. J Intellect Disabil Res. 2014;58:979-91. Demonstrates a relationship between sexual and violent offending and maltreatment in juveniles with intellectual disability.

15. Hulbert-Williams L, Hastings R, Owen DM, et al. Exposure to life events as a risk factor for psychological problems in adults with intellectual disabilities: a longitudinal design. J Intellect Disabil Res. 2014;58(1):48-60.

16. Wigham S, Taylor JL, Hatton C. A prospective study of the relationship between adverse life events and trauma in adults with mild to moderate intellectual disabilities. J Intellect Disabil Res. 2013. doi:10.1111/jir.12107.

17. Dekel S, Solomon Z, Horesh D, Ein-Dor T. Posttraumatic stress disorder and depressive symptoms: joined or independent sequelae of trauma? J Psychiatr Res. 2014;54:64-9.

18. Wigham S, Hatton C, Taylor JL. The Lancaster and Northgate Trauma Scales (LANTS): the development and psychometric properties of a measure of trauma for people with mild to moderate intellectual disabilities. Res Dev Disabil. 2011;32(6):2651-9.

19. Hastings R. Running to catch up: rapid generation of evidence for interventions in learning disability services. Br J Psychiatry. 2013;203:245-6.

20. McElvaney R. Disclosure of child sexual abuse: delays, nondisclosure and partial disclosure. What the research tells us and implications for practice. Child Abuse Rev. 2013. doi:10.1002/ car.2280.

21. Friedman MJ, Resick PA, Bryant RA, Brewin CR. Considering PTSD for DSM-5. Depression Anxiety. 2011;28(9):750-69.

22. Mol SSL, Arntz A, Metsemakers JFB, et al. Symptoms of posttraumatic stress disorder after non-traumatic events: evidence from an open population study. Br J Psychiatry. 2005;186(6):494-9.

23. Spitzer RL, First MB, Wakefield JC. Saving PTSD from itself in DSM-V. J Anxiety Disord. 2007;21(2):233-41.

24. Weathers FW, Keane TM. The criterion A problem revisited: controversies and challenges in defining and measuring psychological trauma. J Trauma Stress. 2007;20(2):107-21.

25. McDonald MK, Borntrager CF, Rostad W. Measuring trauma: considerations for assessing complex and non-PTSD Criterion A childhood trauma. J Trauma Dissociation. 2014;15(2):184-203. 
26. Barol BI, Seubert A. Stepping stones: EMDR treatment of individuals with intellectual and developmental disabilities and challenging behavior. J EMDR Pract Res. 2010;4(4):156-69.

27. Focht-New G, Clements PT, Beth B, Faulkner MJ, Service KP. Persons with developmental disabilities exposed to interpersonal violence and crime: strategies and guidance for assessment. Perspect Psychiatr Care. 2008;44(1):3-13.

28. Mevissen L, Lievegoed R, Seubert A, De Jongh A. Do persons with intellectual disability and limited verbal capacities respond to trauma treatment? J Intellect Dev Disabil. 2011;36(4):278-83.

29. Mevissen L, De Jongh A. PTSD and its treatment in people with intellectual disabilities: a review of the literature. Clin Psychol Rev. 2010;30(3):308-16.

30. Logie R. EMDR-more than just a therapy for PTSD? Psychologist. 2014;27(7):512-6.

31. Hall JC, Jobson L, Langdon PE. Measuring symptoms of posttraumatic stress disorder in people with intellectual disabilities: the development and psychometric properties of the Impact of Event Scale-Intellectual Disabilities (IES-IDs). Br J Clin Psychol. 2014;53:315-32.

32. Bakken TL, Kildahl AN, Gjersoe V, et al. Identification of PTSD in adults with intellectual disabilities in five patients in a specialised psychiatric inpatient unit. Adv Ment Health Intellect Disabil. 2014;8(2):91-102. Describes assessment of PTSD in individuals with moderate to severe intellectual disability.

33. Ardino V. Offending behaviour: the role of trauma and PTSD. Eur J Psychotraumatol. 2012;3:10.

34. Stappenbeck CA, Hellmuth JC, Simpson T, Jacupcak M. The effects of alcohol problems, PTSD, and combat exposure on nonphysical and physical aggression among Iraq and Afghanistan war veterans. Psychol Trauma Theory Res Pract Policy. 2014;6(1):65.

35. Cooper SA, Smiley E, Jackson A, et al. Adults with intellectual disabilities: prevalence, incidence and remission of aggressive behaviour and related factors. J Intellect Disabil Res. 2009;53(3):217-32.

36. Krause-utz A, Elzinga BM, Oei NY, Paret C, Niedtfeld I, Spinhoven $\mathrm{P}$, et al. Amygdala and dorsal anterior cingulate connectivity during an emotional working memory task in borderline personality disorder patients with interpersonal trauma history. Front Hum Neurosci. 2014;8:848.

37. Chinn D, Hall I, Ali A, Hassell H, Patkas I. Psychiatric in-patients away from home: accounts by people with intellectual disabilities in specialist hospitals outside their home localities. J Appl Res Intellect Disabil. 2011;24(1):50-60.

38. Gaskin CJ, McVilly KR, McGillivray KA. Initiatives to reduce the use of seclusion and restraints on people with developmental disabilities: a systematic review and quantitative synthesis. Res Dev Disabil. 2013;34(11):3946-61.

39. Paterson B, McIntosh I, Wilkinson D, McComish, Smith I. Corrupted cultures in mental health inpatient settings. Is restraint reduction the answer? J Psychiatr Ment Health Nurs. 2013;20(3): 228-35.

40. Deb S, Unwin G, Deb T. Characteristics and the trajectory of psychotropic medication use in general and antipsychotics in particular among adults with an intellectual disability who exhibit aggressive behaviour. J Intellect Disabil Res. 2014;59(1):11-25.

41. Romeo R, Knapp M, Tyrer P, Crawford M, Oliver-Africano P. The treatment of challenging behaviour in intellectual disabilities: costeffectiveness analysis. J Intellect Disabil Res. 2009;53(7):633-43.

42. Glover G, Bernard S, Branford D, Holland A, Strydom A. Use of medication for challenging behaviour in people with intellectual disability. Br J Psychiatry. 2014;205(1):6-7.

43. Matson JL, Mahan S. Antipsychotic drug side effects for persons with intellectual disability. Res Dev Disabil. 2010;31(6):1570-6.

44. Hellenbach M, Brown M, Karatzias T, Robinson R. Psychological interventions for women with intellectual disabilities and forensic care needs: a systematic review of the literature. J Intellect Disabil
Res. 2014. doi:10.1111/jir.12133. Highlights the need for more research into treatment interventions for women with intellectual disability in forensic settings.

45. Mendes P, Snow P. The needs and experiences of young people with a disability transitioning from out-of-home care: the views of practitioners in Victoria, Australia. Child Youth Serv Rev. 2014;36: 115-23.

46. Larkin H, Felliti VJ, Anda RF. Social work and adverse childhood experiences research: implications for practice and health policy. Soc Work Pub Health. 2014;29(1):1-16.

47. Matthews KA, Chang YF, Thurston RC, Bromberger JT. Child abuse is related to inflammation in mid-life women: role of obesity. Brain Behav Immun. 2014;36:29-34.

48. Melville CA, Cooper SA, Morrison J, et al. The prevalence and determinants of obesity in adults with intellectual disabilities. J Appl Res Intellect Disabil. 2008;21(5):425-37.

49. Carroll Chapman SL, L-T W. Substance abuse among individuals with intellectual disabilities. Res Dev Disabil. 2012;33(4):1147-56.

50. Pezzoni V, Kouimtsidis C. Screening for alcohol misuse within people attending a psychiatric intellectual disability community service. J Intellect Disabil Res. 2014. doi:10.1111/jir.12168.

51. Didden R, Embregts P, van der Toorn N, Laarhoven N. Substance abuse, coping strategies, adaptive skills and behavioral and emotional problems in clients with mild to borderline intellectual disability admitted to a treatment facility: a pilot study. Res Dev Disabil. 2009;30(5):927-32.

52. Hermans H, Beekman ATF, Evenhuis HM. Prevalence of depression and anxiety in older users of formal Dutch intellectual disability services. J Affect Disord. 2013;144:94-100.

53. Ludi E, Ballard ED, Greenbaum R, et al. Suicide risk in youth with intellectual disability: the challenges of screening. J Dev Behav Pediatr. 2012;33(5):431-40.

54. Savage A, McConnell D, Emerson E, Llewellyn G. Disabilitybased inequity in youth subjective well-being: current findings and future directions. Disabil Soc. 2014;29(6):877-92.

55. Matson JL, Belva BC, Hattier MA, Matson ML. Scaling methods to measure psychopathology in persons with intellectual disabilities. Res Dev Disabil. 2012;33(2):549-62.

56. Hermans H, Evenhuis HM. Characteristics of instruments screening for depression in adults with intellectual disabilities: systematic review. Res Dev Disabil. 2010;31(6):1109-20.

57. Hermans H, van der Pas FH, Evenhuis HM. Instruments assessing anxiety in adults with intellectual disabilities: a systematic review. Res Dev Disabil. 2011;32(3):861-70.

58. Allen D, Langthorne P, Tonge B, Emerson E, McGill P, Fletcher R, et al. Towards the prevention of behavioural and psychiatric disorders in people with intellectual disabilities. J Appl Res Intellect Disabil. 2013;26(6):501-14.

59. Willner P, Rose J, Jahoda A, et al. Group-based cognitive-behavioural anger management for people with mild to moderate intellectual disabilities: cluster randomised controlled trial. $\mathrm{Br} \mathrm{J}$ Psychiatry. 2013;203(4):288-96.

60. Lindsay WR, Tinsley $\mathrm{S}$, Beail N, et al. A preliminary controlled trial of a trans-diagnostic programme for cognitive behaviour therapy with adults with intellectual disability. J Intellect Disabil Res. 2014. doi:10.1111/jir.12145.

61. Tranah T, Nicholas J. Interventions for young people with intellectual disabilities who commit arson. Adv Ment Health Intellect Disabil. 2013;7(2):72-81.

62. McGillivray JA, Kershaw MM. The impact of staff initiated referral and intervention protocols on symptoms of depression in people with mild intellectual disability. Res Dev Disabil. 2013;34(2): 730-8.

63. Pert C, Jahoda A, Stenfert Kroese B, et al. Cognitive behavioural therapy from the perspective of clients with mild intellectual 
disabilities: a qualitative investigation of process issues. $\mathrm{J}$ Intellect Disabil Res. 2013;57(4):359-69.

64. Idusohan-Moizer H, Sawicka A, Dendle J, Albany M. Mindfulnessbased cognitive therapy for adults with intellectual disabilities: an evaluation of the effectiveness of mindfulness in reducing symptoms of depression and anxiety. J Intellect Disabil Res. 2013. doi: 10.1111/jir.12082.

65. Maercker A, Horn AB. A socio-interpersonal perspective on PTSD: the case for environments and interpersonal processes. Clin Psychol Psychother. 2013;20(6):465-81.

66. Charuvastra A, Cloitre M. Social bonds and posttraumatic stress disorder. Annu Rev Psychol. 2008;59:301-28.
67. Keesler JM. A call for the integration of trauma-informed care among intellectual and developmental disability organizations. J Policy Pract Intellect Disabil. 2014;11(1):34-42.

68. Bradley E, Sinclair L, Greenbaum R. Trauma and adolescents with intellectual disabilities: interprofessional clinical and service perspectives. J Child Adolesc Trauma. 2012;5:33-46.

69. Hollomotz A. Disability, oppression and violence: towards a sociological explanation. Sociology. 2013;47(3):477-93.

70. Hutchinson LM, Hastings RP, Hunt PH, et al. Who's challenging who? Changing attitudes towards those whose behaviour challenges. J Intellect Disabil Res. 2014;58(2):99-109. 